\title{
Anticorps \\ anti-CD38 \\ dans le myélome multiple
}

> La forte expression de la molécule CD38 par les cellules plasmocytaires ainsi que son rôle biologique dans la régulation de l'adhérence et la migration cellulaire, avec des fonctions de signalisation, a conduit au développement d'anticorps spécifiques pour le traitement de patients atteints de myélome multiple (MM). Ces anticorps induisent en effet la mort des cellules de myélome multiple par des mécanismes de lyse cellulaire dépendante du complément (CDC), de cytotoxicité cellulaire dépendante des anticorps ( $A D C C)$, de phagocytose cellulaire dépendant des anticorps ( $A D C P)$, mais aussi par des mécanismes directs d'induction de mort cellulaire. Ils ont de plus des effets immunomodulateurs liés à l'élimination de cellules immunitaires immunosuppressives qui expriment également CD38. Bien qu'ayant des actions variables par rapport à ce registre d'activité si on les compare entre eux, les anticorps anti-CD38 ont démontré une activité clinique significative, seuls ou en combinaison avec diverses molécules, chez les patients atteints de MM. Ils contribueront sans aucun doute à des progrès majeurs pour la prise en charge thérapeutique des patients atteints de MM. <

Le traitement du myélome multiple (MM) a profondément évolué au cours des dernières décennies avec l'arrivée de nouvelles molécules qui ont considérablement amélioré la prise en charge thérapeutique des patients [1]. Ces progrès thérapeutiques ont vu le jour à la suite d'une meilleure compréhension de la biologie de la maladie, qui a permis d'identifier de nouvelles voies dérégulées et de nouvelles cibles thérapeutiques. Un des changements majeurs a ainsi été l'introduction des inhibiteurs du protéasome $(\mathrm{Pi})$ et d'agents immunomodulateurs (IMiD) qui ont amélioré très significativement la survie sans progression et la survie globale des patients atteints de MM. Plus récemment, des anticorps ciblant des antigènes membranaires exprimés par les cellules de MM ont été
Jérôme Moreaux ${ }^{1-3}$

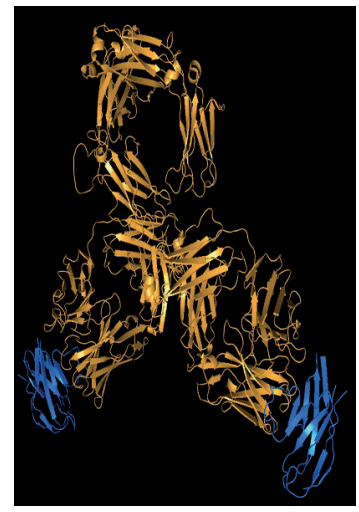

${ }^{1} \mathrm{CHU}$ Montpellier, Département d'hématologie biologique, Montpellier, France.

${ }^{2}$ IGH, CNRS, Univ Montpellier, France.

${ }^{3}$ Univ Montpellier, UFR de Médecine, Montpellier, France. jerome.moreaux@igh.cnrs.fr

développés [2]. Parmi les antigènes ciblés par ces anticorps, la molécule CD38 présente un profil d'expression et des fonctions biologiques qui ont conduit au développement d'anticorps spécifiques pour le traitement des patients atteints de MM [3]. CD38 est une glycoprotéine membranaire de type 2 qui joue un rôle dans la régulation de la migration et de l'adhérence des cellules, avec des fonctions de signalisation. Cette molécule présente une activité ecto-enzymatique qui participe au catabolisme du NAD+ et NADP (nicotinamide adénine dinucléotide réduit/phosphate), contrôlant ainsi le niveau de NAD+ dans le cytoplasme : l'inhibition de CD38 est à l'origine d'une augmentation du niveau intracellulaire de NAD+. Plus récemment, un rôle de CD38 a été décrit dans la production d'adénosine, connue pour présenter des effets immunosuppresseurs $[4,5]$.

CD38 est fortement exprimé par les plasmocytes, mais aussi par d'autres types cellulaires comme les cellules lymphoïdes et myéloïdes, les hématies et les plaquettes et par des cellules non hématopoïétiques. Les cellules plasmocytaires sont néanmoins les cellules qui expriment le plus fortement CD38, suivis des cellules NK (natural killer) et des lymphocytes B et T. Le promoteur de CD38 présente des îlots CpG et un site de fixation pour le facteur de transcription SPl. En amont, des sites potentiels pour les facteurs de transcription TCF-1 $\alpha$ (T-cell factor 1 ), IRFl (interferon regulatory factor 1) et NF-IL6 (nuclear factor of interleukin 6) ont été décrits.

Le daratumumab a été le premier anticorps anti-CD38 approuvé pour le traitement des patients atteints de MM, en monothérapie et en combinaison. D'autres, tels que l'isatuximab, le M0R202 et le TAK079 , sont actuellement en évaluation clinique. Dans cette revue, nous discuterons des mécanismes d'action des anticorps anti-CD38, de leur efficacité clinique en monothérapie ou en combinaison avec d'autres agents thérapeutiques, ainsi que des mécanismes de résistance. 


\section{Mécanismes d'action des anticorps anti-CD38}

Les anticorps anti-CD38 induisent la mort des cellules tumorales par des mécanismes de $C D C$, d'ADCC, et d'ADCP $[6,7]$. Les différents anticorps anti-CD38 présentent cependant des potentiels variables d'induction de ces effets de CDC, d'ADCC et d'ADCP $[8,9]$.

Le premier anticorps anti-CD38 qui fut testé cliniquement a été le daratumumab. Cet anticorps a été sélectionné parmi un panel de 42 anticorps, en raison de sa capacité unique à induire une cytotoxicité dépendante du complément (CDC) [8]. Il induit aussi la mort des cellules de MM par ADCC. La dérégulation des fonctions enzymatiques de CD38 induite par le daratumumab pourrait aussi contribuer à son effet antitumoral [10].

L'isatuximab et le MOR202 ont également des fonctions de CDC, d'ADCC et d'ADCP $[9,10]$. L'isatuximab a été sélectionné en fonction de sa capacité à induire une mort des cellules de MM indépendante des cellules immunitaires effectrices mais aussi dans un contexte tumoral de cellules mutées sur le gène $\operatorname{TP} 53[9,11]$. Aucun lien n'a été établi à ce jour entre les différences fonctionnelles des anticorps anti-CD38 et leur efficacité thérapeutique (Figure 1).

En parallèle des mécanismes d'action classiques qui reposent sur sa région $\mathrm{Fc}$, le daratumumab présente aussi des effets immunomodulateurs qui sont liés à l'élimination de cellules immunitaires immunosuppressives, telles que les lymphocytes T régulateurs, les lymphocytes $B$ régulateurs et les cellules myéloïdes suppressives, aboutissant à une réponse anti-tumorale plus efficace [12]. En réponse au traitement par les anticorps anti-CD38, les patients présentent ainsi des niveaux d'activation des lymphocytes T CD8 $8^{+}$plus importants, associés à une production plus importante de granzyme B. À noter que des travaux ont également montré que l'isatuximab ciblait directement les lymphocytes T régulateurs exprimant CD38 [13].

\section{Utilisation des anticorps anti-CD38 en monothérapie ou en combinaison dans le MM}

Le daratumumab a été testé initialement dans le contexte de patients atteints de MM réfractaires ou résistants aux agents immunomodulateurs et/ou aux inhibiteurs du protéasome, patients qui présentaient un pronostic péjoratif $[14,15]$. Les études qui ont été réalisées ont conduit à son agrément par les autorités américaine (FDA, food and drug administration) et européenne (EMA, European medicines agency) pour le traitement des patients atteints de $M M$ en rechute ou réfractaires. $L a$ dose de $16 \mathrm{mg} / \mathrm{kg}$ de daratumumab a démontré une saturation rapide et durable de CD38 dans la majorité des cas [16]. Chez ces patients, l'anticorps utilisé en monothérapie a été associé à un taux de réponse de $31 \%$ avec une médiane de survie sans progression de 4 mois, et une médiane de survie globale de 20 mois [17]. Le daratumumab présente néanmoins des toxicités, mais celles-ci restent gérables et pourraient être diminuées en modifiant les modes d'administration [17, 18]. Par la suite, d'autres anticorps, incluant l'isatuximanb et le MOR202, ont démontré leur efficacité dans le traitement des patients atteints de MM réfractaires ou résistants aux traitements conventionnels [19, 20].
Les associations thérapeutiques représentent en fait une stratégie efficace pour améliorer la prise en charge thérapeutique des patients atteints de MM, en raison de la grande hétérogénéité de la maladie. Ainsi, les anticorps anti-CD38 apparaissent particulièrement intéressants lorsqu'ils sont associés à d'autres agents thérapeutiques utilisés dans le traitement du MM. La combinaison anticorps anti-CD38 et agents immunomodulateurs, tels que le lénalidomide et le pomalidomide, augmente ainsi significativement l'ADCC exercée par les cellules NK [3, 11, 21-23]. Ces observations ont été à l'origine d'essais cliniques combinant les anticorps anti-CD38 aux IMiD. Un essai randomisé de phase III (POLLUX), comparant un traitement par daratumumab, lénalidomide et dexaméthasone avec un traitement lénalidomide/ dexaméthasone chez des patients ayant déjà reçu au moins une ligne de traitement, a montré une survie sans progression significativement plus importante chez les patients ayant reçu le daratumumab [24]; un nombre significativement plus important de patients traités par le daratumumab présentant une maladie résiduelle négative a également été rapporté. Cette amélioration de survie sans progression est également observée chez les patients présentant des anomalies cytogénétiques de mauvais pronostic (comme la délétion del17p ou les translocations $\mathrm{t}(4 ; 14)$ et $\mathrm{t}(14 ; 16))$. Le daratumumab en association n'abolit cependant pas complétement le pronostic péjoratif de ces anomalies cytogénétiques. Cette association a néanmoins été approuvée par la FDA et l'EMA pour le traitement des patients atteints de MM ayant déjà reçu au moins une ligne thérapeutique.

D'autres AcM anti-CD38, dont l'isatuximab et le MOR202, ont également été testés en combinaison avec le lénalidomide et la dexaméthasone et ont montré une synergie clinique avec ces deux molécules [19, 25]. Une association d'anticorps anti-CD38 avec le pomalidomide et la dexaméthasone a également démontré une efficacité clinique $[19,26,27]$ et une synergie entre anticorps anti-CD38 et inhibiteurs du protéasome a été rapportée dans des études précliniques [3,22].

Les anticorps anti-CD38 peuvent être combinés efficacement avec le carfilzomib, un inhibiteur du protéasome de deuxième génération [28]; le daratumumab, en combinaison avec le lénalidomide et la dexaméthasone, a démontré son efficacité clinique chez les patients atteints de MM ayant déjà reçu au moins une ligne de traitement [29].

Les effets secondaires associés aux AcM anti-CD38 comprennent majoritairement des réactions de grade 1/2 (les toxicités de grade $1 / 2$ sont des toxicités légères à modérées) liées à la perfusion que l'on retrouve chez environ $50 \%$ des patients $[17,24,29]$. 


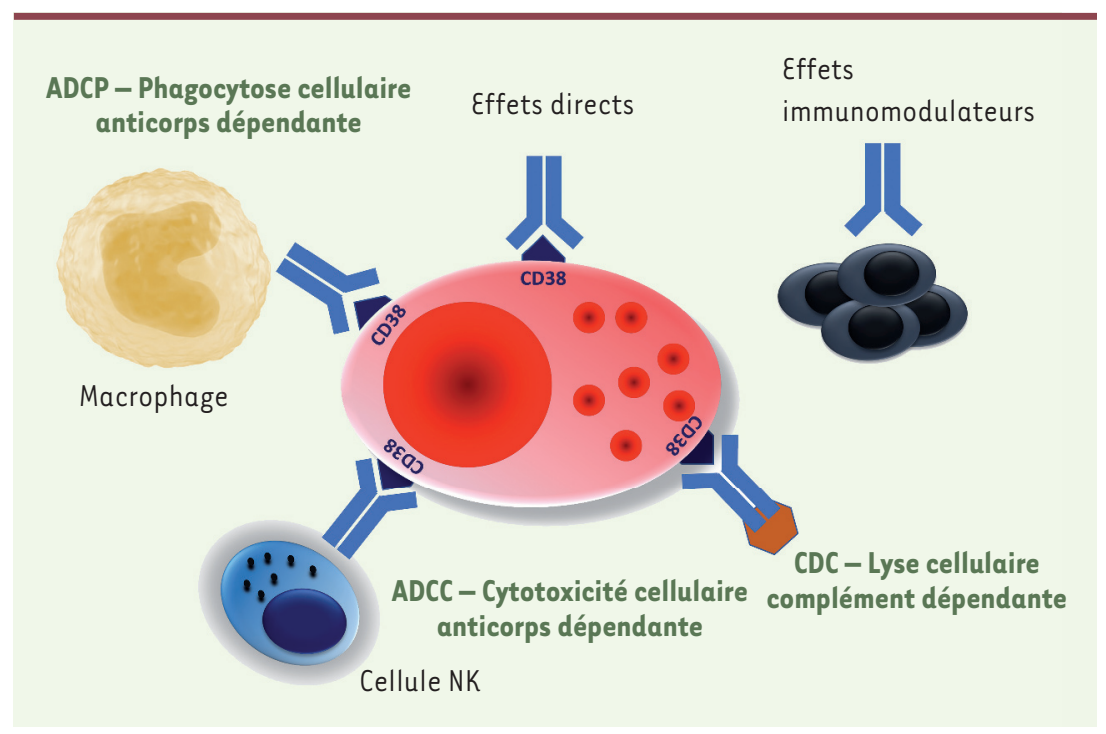

Figure 1. Différences fonctionnelles des anticorps anti-CD38 et efficacité thérapeutique.

cytose dépendant des anticorps anti-CD38 [36] en interagissant avec le régulateur négatif SIRP $\alpha$ (signal regulatory protein $\alpha)^{1}$. Le ciblage de cette interaction pourrait donc accroître l'activité cytolytique dépendant des anticorps anti-CD38. De faibles doses de cyclophosphamide peuvent par ailleurs inhiber l'expression de CD47 par les cellules tumorales et augmenter l'expression des récepteurs pour la région $\mathrm{Fc}$ des IgG par les macrophages, potentialisant ainsi l'ADCP due au daratumumab [37, 38]. L'induction de l'expression du points de contrôle immunitaire PD-1 (programmed cell death-1) et de son ligand, PD-Ll (programmed cell deathligand 1), peut également contribuer au développement de résistances aux fonctions immunomodulatrices des anticorps

\section{Mécanismes de résistance}

L'efficacité de I'ADCC et de la CDC par le daratumumab est corrélée au niveau d'expression de CD38 par les cellules tumorales. En effet, le traitement par daratumumab est moins efficace contre les cellules de MM présentant une faible expression de CD38 [30, 31]. Une faible expression de $\operatorname{CD} 38$ pourrait donc être associée à une résistance primaire des cellules de MM au traitement. L'acide trans-rétinoïque (ATRA) permet d'augmenter l'expression de CD38 par les cellules de $M M$, sans effet sur leur viabilité [30]. Le panobinostat, un inhibiteur d'histone désacétylase (HDAC), conduit également à une augmentation de l'expression de CD38 par les cellules de MM, ce qui permet une meilleure efficacité du daratumumab contre ces cellules [32].

Une augmentation significative de l'expression des protéines inhibitrices du complément CD55 (ou DAF, pour decay accelerating factor) et CD59 (ou protectine) a été observée au moment de la progression de la maladie chez les patients sous traitement par daratumumab. Chez certains patients, plusieurs sous-populations de cellules tumorales exprimant CD55 et CD59 à des niveaux variables co-existent [31]. Durant le traitement, la pression de sélection exercée par le daratumumab conduit à une sélection de cellules tumorales exprimant de hauts niveaux de ces deux inhibiteurs du complément et qui deviennent alors résistantes à son action [19]. L'ATRA induit également une inhibition de l'expression de CD55 et de CD59 par les cellules de MM avec une amélioration de la CDC via les anticorps anti-CD38 [31]. Les anticorps antiCD38 entraînent également une réduction rapide du nombre de cellules NK qui expriment fortement CD38 [33]. Cet effet indésirable peut être contrecarré par l'administration de cellules NK amplifiées ex vivo [34]. Les cellules de MM sont aussi protégées de l'ADCC induite par le daratumumab par les cellules stromales médullaires via l'induction de l'expression par les cellules tumorales de protéines anti-apoptotiques, telles que Mcl-1 (myeloid cell leukemia-1) et la survivine [35]. De même, l'expression de CD47 par les cellules de MM inhibe leur phago-
anti-CD38 [39, 40]. Des données précliniques montrent que l'association d'anticorps anti-PD-1/PD-Ll à des anticorps anti-CD38 pourraient présenter un intérêt thérapeutique afin d'améliorer la prise en charge des patients $[41,42]$.

\section{Conclusion}

Seuls ou en combinaison avec des agents immunomodulateurs et des inhibiteurs du protéasome, les anticorps anti-CD38 ont montré une importante activité clinique chez les patients atteints de MM, incluant les patients réfractaires ou en rechute. Le développement de différents anticorps anti-CD38 ayant des fonctions effectrices différentes présente un intérêt pour appréhender l'apparition de résistances. D'autres anticorps thérapeutiques spécifiques de cibles différentes également exprimées par les cellules de MM sont en développement clinique. Le développement de l'immunothérapie dans l'arsenal thérapeutique permettra des progrès majeurs dans la prise en charge thérapeutique des patients atteints de MM. $\diamond$

\section{SUMMARY}

CD38 antibodies in multiple myeloma

The high CD38 expression by plasma cells together with the biological functions of CD38 resulted in the development of CD38 antibodies for the treatment of multiple myeloma (MM) patients. The cytolytic activity of CD38

${ }^{1}$ CD47 est exprimé par les cellules de différentes lignées cancéreuses. SIRP $\alpha$ est un récepteur inhibiteur présent sur les macrophages. L'interaction de CD47 avec SIRP $\alpha$ active les phosphatases SHP-1,-2 (Src homology region 2 domain-containing phosphatase-1 et -2) qui inhibent la phagocytose. 
antibodies is mediated by complement-dependent cytoxicity (CDC), antibody-dependent cell-mediated cytotoxicity (ADCC), antibodydependent cellular phagocytosis ( $A D C P$ ), direct cell death effects and immunomodulatory effects. CD38 antibodies have demonstrated their clinical benefit as single agent or in combination for the treatment of multiple myeloma patients and will contribute to further improvement in the outcome of MM patients. $\diamond$

\section{LIENS D'INTÉRÊT}

L'auteur déclare n'avoir aucun lien d'intérêt concernant les données publiées dans cet article.

\section{RÉFÉRENCES}

1. Barlogie B, Mitchell A, van Rhee F, et al. Curing myeloma at last: defining criteria and providing the evidence. Blood $2014 ; 124: 3043-51$.

2. van de Donk NW, Moreau P, Plesner T, et al. Clinical efficacy and management of monoclonal antibodies targeting CD38 and SLAMF7 in multiple myeloma. Blood 2016 ; $127: 681-95$.

3. van de Donk N, Richardson PG, Malavasi F. CD38 antibodies in multiple myeloma: back to the future. Blood $2018 ; 131: 13-29$.

4. Munoz P, Mittelbrunn M, de la Fuente $H$, et al. Antigen-induced clustering of surface CD38 and recruitment of intracellular CD38 to the immunologic synapse. Blood 2008; $111: 3653-64$

5. Howard M, Grimaldi JC, Bazan JF, et al. Formation and hydrolysis of cyclic ADP-ribose catalyzed by lymphocyte antigen CD38. Science $1993 ; 262: 1056-9$.

6. van de Donk N, Usmani SZ. CD38 Antibodies in multiple myeloma: Mechanisms of action and modes of resistance. Front Immunol $2018 ; 9: 2134$.

7. van de Donk NW, Janmaat ML, Mutis T, et al. Monoclonal antibodies targeting CD38 in hematological malignancies and beyond. Immunol Rev 2016 ; 270 : 95-112.

8. de Weers M, Tai YT, van der Veer MS, et al. Daratumumab, a novel therapeutic human CD38 monoclonal antibody, induces killing of multiple myeloma and other hematological tumors.J Immunol $2011 ; 186: 1840-8$.

9. Deckert J, Wetzel MC, Bartle LM, et al. SAR650984, a novel humanized CD38-targeting antibody, demonstrates potent antitumor activity in models of multiple myeloma and other CD38+ hematologic malignancies. Clin Cancer Res 2014 ; $20: 4574-83$.

10. Lammerts van Bueren J, Jakobs D, Kaldenhoven N, et al. Direct in vitro comparison of daratumumab with surrogate analogs of CD38 antibodies MOR03087, SAR650984 and Ab79. Blood $2014 ; 124: 3474-$

11. Jiang $\mathrm{H}$, Acharya C, An G, et al. SAR650984 directly induces multiple myeloma cell death via lysosomal-associated and apoptotic pathways, which is further enhanced by pomalidomide. Leukemia 2016 ; 30 : 399-408.

12. Krejcik J, Casneuf T, Nijhof IS, et al. Daratumumab depletes CD38+ immune regulatory cells, promotes T-cell expansion, and skews T-cell repertoire in multiple myeloma. Blood $2016 ; 128: 384-94$.

13. Feng $X$, Zhang L, Acharya $C$, et al. Targeting CD38 suppresses Induction and function of $T$ regulatory cells to mitigate immunosuppression in multiple myeloma. Clin Cancer Res $2017 ; 23$ : 4290-300.

14. Lokhorst HM, Plesner T, Laubach JP, et al. Targeting CD38 with daratumumab monotherapy in multiple myeloma. $N$ Engl J Med 2015 ; 373 : 1207-19.

15. Lonial S, Weiss BM, Usmani SZ, et al. Daratumumab monotherapy in patients with treatmentrefractory multiple myeloma (SIRIUS): an open-label, randomised, phase 2 trial. Lancet 2016 ; 387 : 1551-60.

16. Clemens PL, Yan X, Lokhorst HM, et al. Pharmacokinetics of daratumumab following intravenous infusion in relapsed or refractory multiple myeloma after prior proteasome inhibitor and immunomodulatory drug treatment. Clin Pharmacokinet 2017 ; 56 : 915-24.

17. Usmani SZ, Weiss BM, Plesner T, et al. Clinical efficacy of daratumumab monotherapy in patients with heavily pretreated relapsed or refractory multiple myeloma. Blood $2016 ; 128: 37-44$

18. Chari A, Nahi H, Mateos M-V, et al. Subcutaneous delivery of daratumumab in patients (pts) with relapsed or refractory multiple myeloma (RRMM): Pavo, an open-label, multicenter, dose escalation phase lb study. Blood $2017 ; 130: 838$.

19. Raab MS, Chatterjee M, Goldschmidt H, et al. A phase I/Ila study of the CD38 antibody MOR202 alone and in combination with pomalidomide or lenalidomide in patients with relapsed or refractory multiple myeloma. Blood $2016 ; 128: 1152$.

20. Richter JR, Martin TG, Vij R, et al. Updated data from a phase II dose finding trial of single agent isatuximab (SAR650984, anti-CD38 mAb) in relapsed/refractory multiple myeloma (RRMM). J Clin Oncol $2016 ; 34: 8005$.

21. Nijhof IS, Groen RW, Noort WA, et al. Preclinical evidence for the therapeutic potential of CD38targeted immuno-chemotherapy in multiple myeloma patients refractory to lenalidomide and bortezomib. Clin Cancer Res 2015 ; 21 : 2802-10.

22. van der Veer MS, de Weers M, van Kessel B, et al. The therapeutic human CD38 antibody daratumumab improves the anti-myeloma effect of newly emerging multidrug therapies. Blood Cancer J $2011 ; 1:$ e4l.

23. van der Veer MS, de Weers M, van Kessel B, et al. Towards effective immunotherapy of myeloma: enhanced elimination of myeloma cells by combination of lenalidomide with the human CD38 monoclonal antibody daratumumab. Haematologica $2011 ; 96: 284-90$.

24. Dimopoulos MA, Oriol A, Nahi H, et al. Daratumumab, lenalidomide, and dexamethasone for multiple myeloma. N Engl J Med 2016 ; 375 : 1319-31.

25. Martin T, Baz R, Benson DM, et al. A phase lb study of isatuximab plus lenalidomide and dexamethasone for relapsed/refractory multiple myeloma. Blood 2017 ; 129 : 3294-303.

26. Chari A, Suvannasankha A, Fay JW, et al. Daratumumab plus pomalidomide and dexamethasone in relapsed and/or refractory multiple myeloma. Blood $2017 ; 130: 974-81$.

27. Richardson PG, Mikhael J, Usmani SZ, et al. Updated results from a phase Ib study of isatuximab plus pomalidomide (Pom) and dexamethasone (dex) in relapsed/refractory multiple myeloma (RRMM). Blood 2017 ; 130 : 1887.

28. Jakubowiak AJ, Chari A, Lonial S, et al. Daratumumab (DARA) in combination with carfilzomib, lenalidomide, and dexamethasone (KRd) in patients (pts) with newly diagnosed multiple myeloma (MMY1001): An open-label, phase lb study. J Clin Oncol $2017 ; 35: 8000$.

29. Palumbo A, Chanan-Khan A, Weisel K, et al. Daratumumab, bortezomib, and dexamethasone for multiple myeloma. N EnglJ Med 2016 ; 375 : 754-66.

30. Nijhof IS, Groen RW, Lokhorst HM, et al. Upregulation of CD38 expression on multiple myeloma cells by all-trans retinoic acid improves the efficacy of daratumumab. Leukemia $2015 ; 29$ : 2039-49.

31. Nijhof IS, Casneuf T, van Velzen J, et al. CD38 expression and complement inhibitors affect response and resistance to daratumumab therapy in myeloma. Blood 2016 ; 128 : 959-70.

32. Garcia-Guerrero $\varepsilon$, Gogishvili T, Danhof S, et al. Panobinostat induces CD38 upregulation and augments the antimyeloma efficacy of daratumumab. Blood 2017 ; 129 : 3386-8.

33. Casneuf T, Xu XS, Adams HC, 3rd, et al. Effects of daratumumab on natural killer cells and impact on clinical outcomes in relapsed or refractory multiple myeloma. Blood Adv 2017 ; 1 : 2105-14.

34. Wang $Y$, Zhang $Y$, Hughes $T$, et al. Fratricide of NK cells in Daratumumab therapy for multiple myeloma overcome by ex vivo-expanded autologous NK cells. Clin Cancer Res 2018 ; 24 : 4006-17.

35. de Haart SJ, Holthof L, Noort WA, et al. Sepantronium bromide (yM155) improves daratumumab-mediated cellular lysis of multiple myeloma cells by abrogation of bone marrow stromal cell-induced resistance. Haematologica 2016 ; 101 : e339-42.

36. van Bommel PE, He Y, Schepel I, et al. CD20-selective inhibition of CD47SIRPalpha don't eat me signaling with a bispecific antibody-derivative enhances the anticancer activity of daratumumab, alemtuzumab and obinutuzumab. Oncoimmunology $2018 ; 7$ : e1386361.

37. Rigalou A, Ryan A, Natoni A, et al. Potentiation of anti-myeloma activity of daratumumab with combination of cyclophosphamide, lenalidomide or bortezomib via a tumor secretory response that greatly augments macrophage-induced ADCP. Blood 2016; $128: 2101$.

38. Naicker S, Rigalou A, McEllistrim C, et al. Patient data supports the rationale of low dose cyclophosphamide to potentiate the anti-myeloma activity of daratumumab through augmentation of macrophage-induced ADCP. Blood $2017 ; 130: 121$.

39. Syn NL, Teng MWL, Mok TSK, Soo RA. De-novo and acquired resistance to immune checkpoint targeting. Lancet Oncol 2017 ; 18 : e731-41.

40. Koyama S, Akbay EA, Li YY, et al. Adaptive resistance to therapeutic PD-1 blockade is associated with upregulation of alternative immune checkpoints. Nat Comm $2016 ; 7: 10501$.

41. Chen L, Byers LA, Ullrich S, et al. CD38 as a novel immune checkpoint and a mechanism of resistance to the blockade of the PD-1/PD-Ll axis. J Clin Oncol $2017 ; 35: 79$.

42. Bezman NA, Kinder M, Jhatakia AD, et al. Antitumor activity associated with dual targeting of CD38 and programmed death-1 (PD-1) pathways in preclinical models. Cancer Res 2018; 78 (abstract 1727).
TIRÉS À PART

J. Moreaux 